\title{
Autooxidation and rearrangement reactions of isoquinolinone derivatives
}

\author{
María de las M. Blanco, María S. Shmidt, and Isabel A. Perillo* \\ Department of Organic Chemistry, Faculty of Pharmacy and Biochemistry, University of Buenos \\ Aires, Junín 956 (1113), Buenos Aires, Argentina \\ E-mail: iperillo@ffyb.uba.ar
}

\begin{abstract}
4-Hydroxy-2-methyl-1-oxo-1,2-dihydroisoquinoline-3-carboxylic acid derivatives $\mathbf{1}$ in solution afford a mixture of the dealkoxycarbonylated product 2 and 4-hydroxy-2-methyl-1,3-dioxo1,2,3,4-tetrahydroisoquinoline-4-carboxylic acid derivatives $\mathbf{3}$. Reactions $\mathbf{1} \rightarrow \mathbf{3}$ involve compounds 1 tautomerization, C-3 hydroxylation and final $\alpha$-ketol type rearrangement. The synthetic utility of this transformation was explored.
\end{abstract}

Keywords: Isoquinolinones, autooxidation, rearrangement, tautomerization, hydroperoxide, hydroxylation

\section{Introduction}

Alkyl 4-hydroxy-2-methyl-1-oxo-1,2-dihydroisoquinoline-3-carboxylates (1, X=OR) are useful polyfunctional synthetic intermediates for the preparation of biologically active compounds. ${ }^{1}$ In the course of our previous research related to the synthesis and properties of isoquinolinones, ${ }^{2}$ we observed that samples of such compounds decomposed in solution. This fact had previously been observed by Lombardino, who reported that methyl ester 1a was unstable, but decomposition products were not isolated. ${ }^{\text {1a }}$

In this work we present the isolation and characterization of final decomposition products of methyl ester 1a, as well as intermediaries leading to them. Structural requirements for such a transformation are evaluated and its synthetic utility is explored. 
<smiles>[X]C(=O)c1c(O)c2ccccc2c(=O)n1C</smiles><smiles>Cn1cc(O)c2ccccc2c1=O</smiles>

2<smiles>[X]C1(O)C(=O)N(C)C(=O)c2ccccc21</smiles>

3

\section{Results and Discussion}

A sample of 4-hydroxy-2-methyl-1-oxo-1,2-dihydroisoquinoline-3-carboxylic acid methyl ester 1a maintained in solution for several weeks, finally transformed into two products which were chromatographically isolated. The most mobile band developed colour with ferric chloride solution. The ${ }^{1} \mathrm{H}-\mathrm{NMR}$ spectrum of the isolated compound showed a broad signal (exchangeable) at $9.2 \mathrm{ppm}$, coherent with an enol hydroxyl, five differentiated signals in the aromatic zone and only one signal corresponding to a lactam $N$-methyl in the aliphatic zone. Such results, together with MS data $\left(\mathrm{M}^{+}: \mathrm{m} / \mathrm{z} 175,100 \%\right)$ lead us to propose the 4-hydroxy-2methyl-1-oxo-1,2-dihydroisoquinoline 2 structure which was unequivocally confirmed by comparison with an authentic sample prepared by acid hydrolysis of $\mathbf{1}^{\text {1a }}$

Elemental analysis and HRMS of compound obtained from the second eluted band fitted with molecular formula: $\mathrm{C}_{12} \mathrm{H}_{11} \mathrm{NO}_{5}$ thus making evident an oxygen atom incorporation in starting compound 1a. By bidimensional heteronuclear correlation spectra (HMQC, HMBC) structure of the product was established as a homophthlimide derivative, namely 4-hydroxy-2methyl-1,3-dioxo-1,2,3,4-tetrahydroisoquinoline-4-carboxylic acid methyl ester 3a. The main correlations observed in the HMBC spectra which account for our proposed structure are shown in Figure 1.

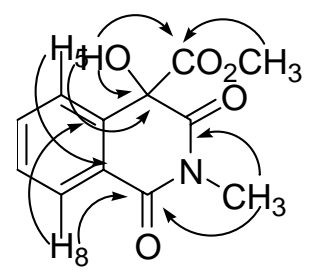

Figure 1. Main correlations in the HMBC spectrum of compound 3a.

This structure is coherent with the observed fragmentation pattern in the mass spectra, showing $\mathrm{m} / \mathrm{z} 190$ as the base ion, resulting from $\mathrm{M}^{+}$. alkoxydecarbonylation (Scheme 1). ${ }^{3}$ 


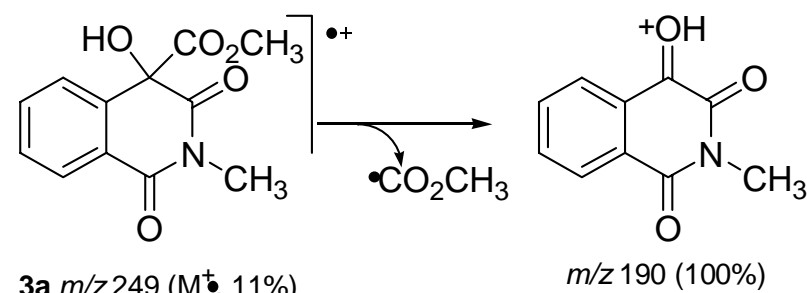

\section{Scheme 1}

\section{Isolation of intermediaries in 1a $\rightarrow$ 3a transformation}

Transformation $\mathbf{1 a} \rightarrow \mathbf{3 a}$ occurs in different solvents, especially in methanol, and yet slowly in the solid state. Several lines of evidences implicate an autooxidation process mediated by free radicals since transformation: i) is accelerated in air bubbled solvent indicating the role of oxygen in the process, ii) does not occur in the absence of light and under strictly deoxigenated conditions, iii) is inhibited by radical scavengers (BTH) and iv) results accelerated by benzoyl peroxide and light.

To ascertain the mechanism of such a profound transformation of isoquinolinone nucleous under mild conditions we made attempts to isolate intermediaries. Thus, a homogeneous sample of methyl ester 1a having a neat enol structure ${ }^{1 \mathrm{a}}$ was dissolved in chloroform, and after brief storage it was periodically analyzed by TLC. Besides products $\mathbf{2}$ and 3a, appearance of three intermediate compounds of low $\mathrm{R}_{\mathrm{f}}$ (chloroform-methanol 9:1), which were consumed by transforming into final products was observed. One of them, eluted under argon atmosphere and isolated as an oil, has the spectroscopic features of dicarbonyl tautomer 4a of starting material (Scheme 2). NMR spectra present resonances which strongly diagnostic its structure. In ${ }^{1} \mathrm{H}-\mathrm{NMR}$ spectrum a singlet at $7.9 \mathrm{ppm}$ (one proton, not exchangeable) is attributed to $\mathrm{H}-3$. In ${ }^{13} \mathrm{C}-\mathrm{NMR}$ spectrum the keto carbonyl (C-4) resonance appears at 188.7 and C-3 (CH, Dept-90) at 90.8 ppm. In agreement with the proposed structure the IR spectra shows a band at $1724 \mathrm{~cm}^{-1}$ related to keto carbonyl stretching. This compound is highly reactive, being very quickly transformed in the presence of oxygen.

Another eluted band afforded a compound of mp 150-152 ${ }^{\circ} \mathrm{C}$ which showed a positive potassium iodide starch test. The HRMS corresponds to a compound of molecular formula $\mathrm{C}_{12} \mathrm{H}_{11} \mathrm{NO}_{6}$ and shows characteristic lost of $\mathrm{OOH}$ and $\mathrm{O}$. The ${ }^{1} \mathrm{H}-\mathrm{NMR}$ spectrum displays an exchangeable signal at $9.90 \mathrm{ppm}$ and a characteristic set of signals for the aromatic protons similar to that of compound 4a, so that hydroperoxide 5a structure was assigned (Scheme 2). According to this ${ }^{13} \mathrm{C}-\mathrm{RMN}$ spectrum shows typical signals of saturated carbon at $94.6(\mathrm{C}-3)$ and keto carbonyl (C-4) at $185.3 \mathrm{ppm}$. 


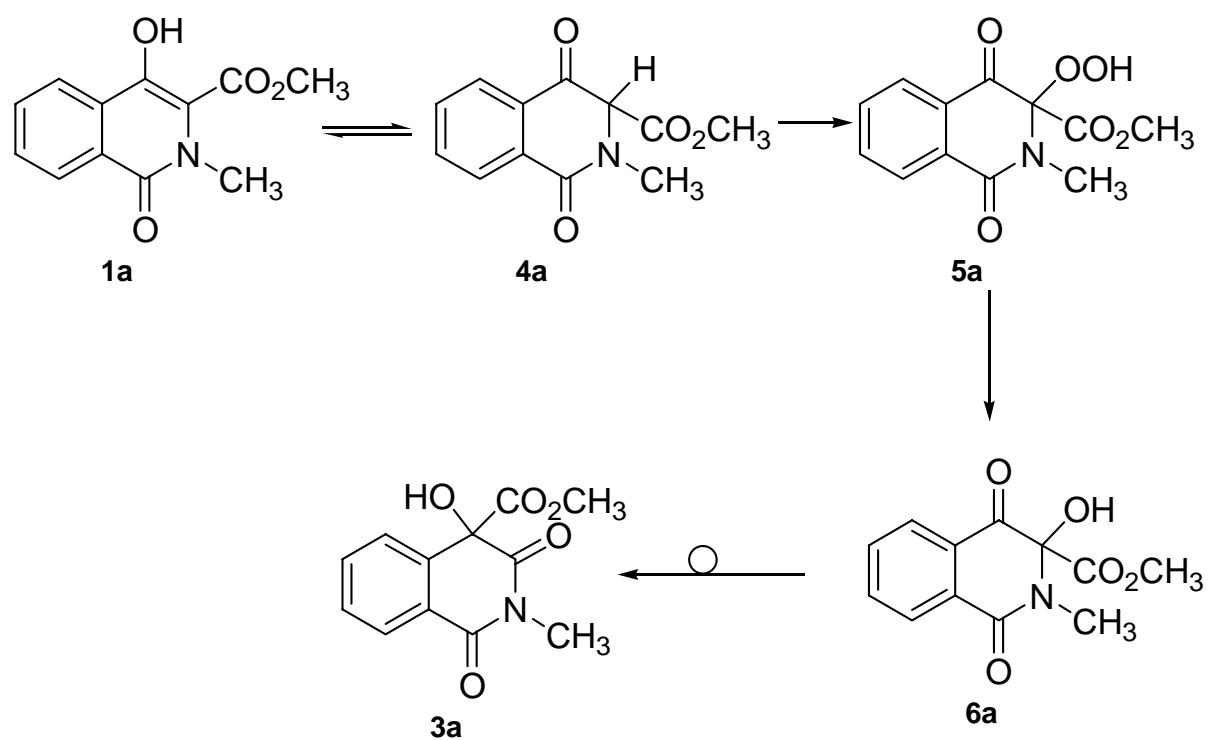

\section{Scheme 2}

The third compound (mp $118-120^{\circ} \mathrm{C}$ ) shows a set of signals for the aromatic protons similar to that of compounds $\mathbf{4 a}$ and $\mathbf{5 a}$ and a exchangeable signal at $4.89 \mathrm{ppm}$ in the ${ }^{1} \mathrm{H}-\mathrm{NMR}$ spectrum. ${ }^{13} \mathrm{C}-\mathrm{NMR}$ shows characteristic signals of saturated carbon at $86.9 \mathrm{ppm}$ (which do not present coupling $\mathrm{C}-\mathrm{H}$ bond) and keto carbonyl at 188.3 (CO-4). HRMS indicates a molecular formula $\mathrm{C}_{12} \mathrm{H}_{11} \mathrm{NO}_{5}$ so that $\mathbf{6 a}$ structure was assigned to this derivative. Structure of compounds $\mathbf{5 a}$ and 6a were confirmed by comparison with authentic samples obtained from 1a (see below, Scheme 4).

To rationalize our experimental results, we presumed that 1a transformation begins with the tautomerization of compound 1a to $\mathbf{4 a}$ followed by C-3 hydroxylation to the hydroxy derivative 6a, probably via the corresponding hydroperoxide 5a as intermediate. Finally, 6a rearrangement leads to the final product 3a (a stable imide) (Scheme 2).

In the following section we analyze features of each step as well as previous literature reports.

The tautomerization 1a $\rightarrow \mathbf{4 a}$ is the key step of the process, from which transformation of compound 1a is unchained. The literature describes some antecedents of 4hydroxyisoquinolinone tautomerization in solution where a shift towards dioxo form is observed. ${ }^{4}$ Afterwards, isolation of two tautomeric forms of 2-(o-acetylaminophenyl)- 4hydroxy-1-oxo-3-phenyl-1,2-dihydroisoquinoline was reported, although structures were only confirmed by IR spectroscopy. ${ }^{5}$

To the best of our knowledge, compound 4a isolated in 1a decomposition represents the first example of isolation of a highly reactive 4-hydroxyisoquinolinone keto tautomer which could be characterized by NMR spectroscopy. The highest speed of 1a $\rightarrow$ 3a transformation in methanol may be related to this step, as it is well known that in keto-enol tautomerism, polar solvents favour the shift to the keto form (the most polar). ${ }^{6}$ 
Tautomer $4 \mathbf{a}$ can also be the origin of compound 2. Easy hydrolysis of $\beta$-ketoester $(\alpha-$ substituted with a strongly withdrawing group), together with spontaneous decarboxylation of the obtained $\beta$-ketoacid ${ }^{7}$ probably accounts for attainment of decarbetoxylated product.

4-Hydroxyisoquinolinone C-3 hydroxylation has precedents in literature. ${ }^{8-11}$. It involves 3acetoxylation of the keto form (generally employing $\mathrm{Hg}(\mathrm{II})$ or $\mathrm{Pb}(\mathrm{IV})$ acetates) with further hydrolysis. $^{12}$

In our case, isolation of hydroperoxide 5a from the reaction mixture as well as reaction conditions mentioned above made us presume that hydroxylation leading to compound 6a is consequence of an autooxidation process mediated by free radicals, in which hydroperoxide $\mathbf{5 a}$ is probably the intermediary. Among literature reports related to this fact, autooxidation of a 2,3dialkyl-4-hydroxy-1,2-dihydroisoquinoline leading to a hydroperoxide and the corresponding alcohol as a result of C-3 oxidation can be mentioned. ${ }^{14}$

Although it is not the objective of our work to continue with the study of the precise mechanism of this reaction, we propose a possible pathway leading to intermediaries $5 \mathbf{a}$ and $6 \mathbf{a}$ (Scheme 3). Taking into account mechanistic antecedents of related reactions, ${ }^{15}$ such process would be initiated by typical free radical sources leading to carbon radical $\mathbf{A}$. This radical reacts with molecular oxygen to form peroxy radical species $\mathbf{B}$ which abstracts hydrogen from the substrate leading to hydroperoxide 5a formation. Finally, formation of hydroxy derivative $\mathbf{6 a}$ can be attributed to homolytic cleavage of the corresponding hydroperoxide to the active alkoxy radical $\mathbf{C}$. Other possible mechanisms cannot be ruled out.<smiles>CC(=O)C1C(=O)c2ccccc2C(=O)N1C</smiles>

$4 a$<smiles>CC(=O)OC1(O)C(=O)c2ccccc2C(=O)N1C</smiles>
$6 a$<smiles>CC(=O)[C@H]1C(=O)c2ccccc2C(=O)N1C</smiles>

A

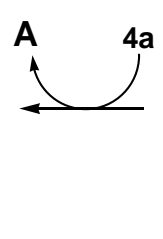<smiles>COC([O])(OC)C1([O-])C(=O)c2ccccc2C(=O)N1C</smiles>

C<smiles>C[13CH]=O</smiles><smiles>COC(=O)C1([O-])C(=O)c2ccccc2C(=O)N1C</smiles>

B

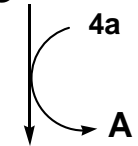

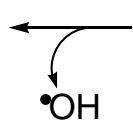<smiles>COC(=O)C1(O)C(=O)c2ccccc2C(=O)N1C</smiles>

\section{Scheme 3}

The rearrangement $6 \mathbf{a} \rightarrow 3 \mathbf{a}$ may be interpreted as a consequence of 1,2-ethoxycarbonyl shift in an $\alpha$-ketol type rearrangement, ${ }^{16}$ favoured by $\mathrm{OH}$ acidity. This type of reactions has been widely studied in aliphatic and carbocyclic compounds, although examples in heterocyclic compounds having $\alpha$-hydroxycarbonyl moiety such as 3-hydroxy-2,4-quinolinediones also exists. ${ }^{17}$ It 
generally occurs by acid or base catalysis although examples of thermally induced reactions have also been reported. In isoquinolone derivatives, literature describes thermal transformation of the 3-hydroxy-1,4-dioxo-3-phenyl-1,2,3,4-tetrahydroisoquinoline into 4-hydroxy-1,3-dioxo-4phenyl-1,2,3,4-tetrahydroisoquinoline ${ }^{8}$ where phenyl is the migratory group. From a mechanistic point of view, it is generally accepted that the fundamental pathway in such rearrangements is an intramolecular migration of a group (which should acquire partial carbanionic character) from the carbinol carbon (C-3) to the potentially electrophylic carbonyl carbon (C-4) together with an intramolecular proton transfer (Figure 2).

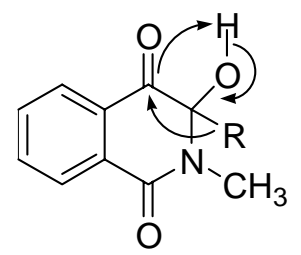

Figure 2. $\alpha$-Ketol type rearrangement of compounds 6 .

However, a mechanism involving a transition state with pronounced dirradicaloid character such as that suggested by Sprecher $^{18}$ cannot be discarded. The facile 1,2-shift of the ethoxycarbonyl group in compound 6a would be comprehensible in terms of the expected stability of the $\mathrm{CO}_{2} \mathrm{R}$ radical. ${ }^{19}$

\section{Scope of the transformation $1 \rightarrow 3$ and synthetic applications}

Although our primary goal was aimed at a better understanding of the possible pathway leading to compound 3a, we were also interested in investigating the scope of the transformation as well as its possible synthetic utility. Thus, a series of isoquinolinones having different structural features was subsequently evaluated.

Ethyl ester $\mathbf{1 b}$ as well as the corresponding $N, N$-diethylamide $\mathbf{1 c}$ show a similar behaviour to that of methyl ester $\mathbf{1 a}$ affording $\mathbf{3 b}$ and $\mathbf{3 c}$ respectively, in approximately 20 days. As it can be expected, compound $\mathbf{3 c}$ shows diastereotopic methylene hydrogens due to the presence of chiral C-4. In contrast to compounds 1a-c, the corresponding $N$-lactam unsubstituted isoquinolinones are stable to the air. Stability of these compounds may be justified taking into account the presence of a resonance assisted hydrogen bonding effect (RAHB) ${ }^{20}$ which involves the enol hydroxyl and the extranuclear carbonyl ${ }^{21}$ (Figure 3 ).

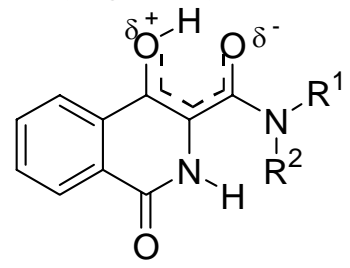

Figure 3. RAHB effect in $N$-lactam unsubstituted isoquinolinones. 
Instead, in isoquinolinones 1a-c, the lactam $N$-methyl steric effect inhibits keto-enol system planarity and hence the RAHB effect, ${ }^{22}$ thus favouring the shift to keto tautomer and further hydroxylation and rearrangement.

In order to evaluate the importance of C-3 hydroxyl in the rearrangement, 3-methoxy derivative 7 was synthesized and tested (Scheme 4). It was obtained by reaction of 1a with NBS in methanol at $-10^{\circ} \mathrm{C}$. This compound is stable at room temperature but it rearranges to 8 by prolonged heating in methanol. The resistance of compound 7 to such rearrangement is probably related to the absence of hydroxyl hydrogen and would evidence a different mechanism from that of hydroxy derivatives 6.

Finally, we turned our attention to explore synthetic utility of $\mathbf{1} \rightarrow \mathbf{3}$ transformation. As it is difficult to fix the best conditions for spontaneous autooxidation and rearrangement of compounds $\mathbf{1}$ in order to obtain acceptable yields of final products $\mathbf{3}$, we explored other variants which allow us the application of this process as a synthetic tool. ${ }^{23}$ Thus, preparation of hydroxy derivative $\mathbf{6} \mathbf{a}$ by reaction of $\mathbf{1 a}$ with NBS at room temperature and its further rearrangement by reflux in water for 2 hs leads to 3a (72\%) (Scheme 4). Alternatively, transformation 1a $\rightarrow$ 3a may be attained via hydroperoxide 5a obtained by catalytic hydroperoxydation of 1 a employing manganese(III) acetate (65\% yield).

Acetoxy derivative 9 (easily obtained by treatment of $\mathbf{1 a}$ with lead(IV) acetate) is also a proper synthetic precursor of 4,4-disubstituted isoquinolinodiones. Compound $\mathbf{9}$ is stable at room temperature but it is transformed to $\mathbf{3 a}$ and $\mathbf{8}$ respectively by heating with water or methanol.

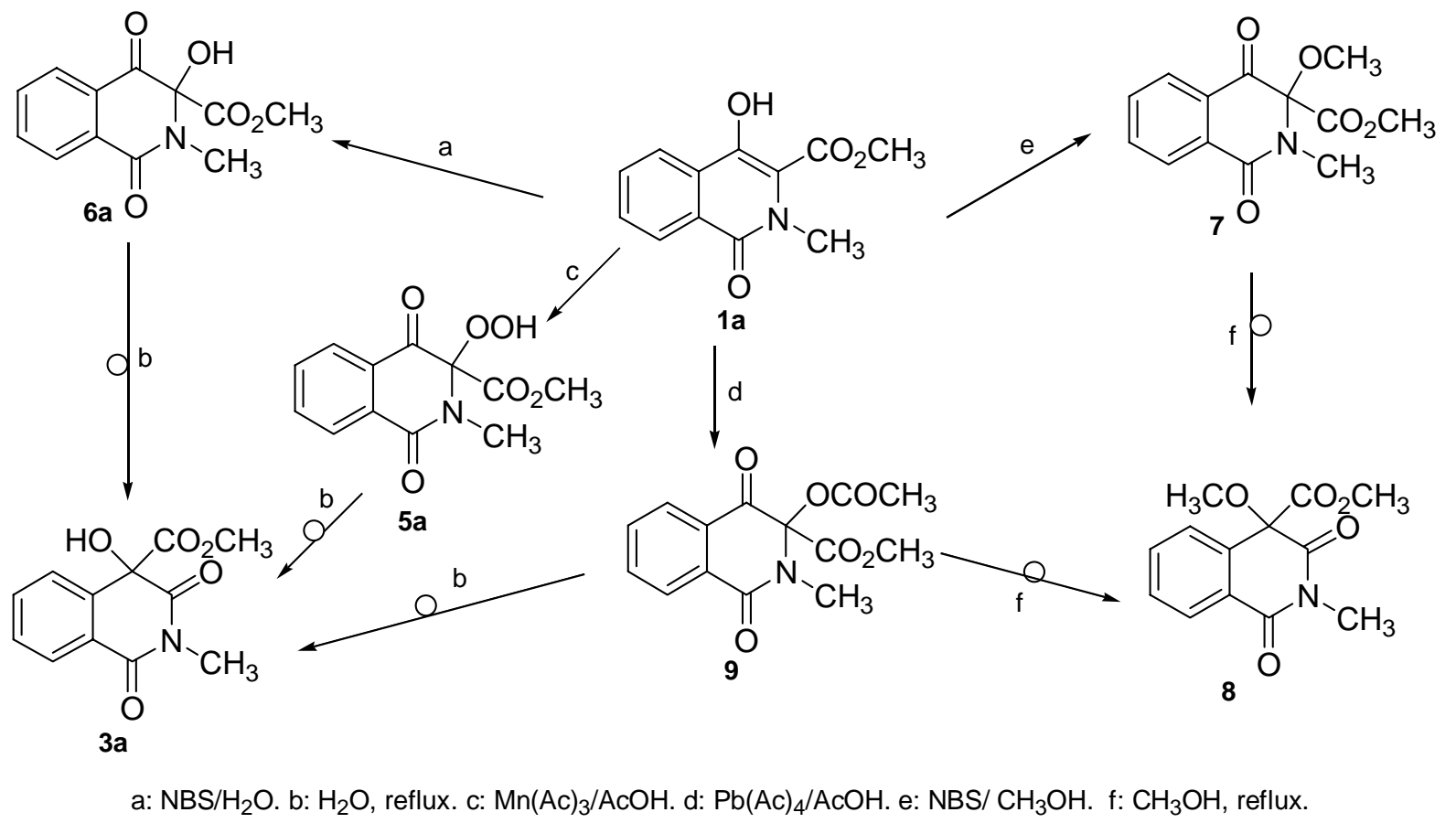

Scheme 4. Chemical transformations of compound 1a with synthetic utility. 


\section{Experimental Section}

General Procedures. Melting points were taken on a Büchi capillary apparatus and are uncorrected. The ${ }^{1} \mathrm{H}$ - and ${ }^{13} \mathrm{C}-\mathrm{NMR}$ spectra were recorded on a Bruker MSL $300 \mathrm{MHz}$ spectrometer employing TMS as internal reference. Deuteriochloroform or DMSO-d6 were used as the solvent, and the standard concentration of the samples for ${ }^{1} \mathrm{H}-\mathrm{NMR}$ was $10 \mathrm{mg} / \mathrm{mL}$ and 25 $\mathrm{mg} / \mathrm{mL}$ for ${ }^{13} \mathrm{C}-\mathrm{NMR}$. Chemical shifts are reported in ppm $(\delta)$ relative to TMS as an internal standard. Deuterium oxide was employed to confirm exchangeable protons (ex). Splitting multiplicities are reported as singlet (s), broad signal (br s), doublet (d), triplet (t), quartet (q), and multiplet (m). Two-dimensional spectra (HMQC, HMBC and ROESY) were recorded with a Bruker AVANCE DRX 300 spectrometer. Electron impact MS were recorded with a GC-MS Shimadzu QP-1000 spectrometer operating at $70 \mathrm{eV}$. Elemental analyses were carried out on a Carlo Erba EA 1108 instrument (Unidad de Microanálisis y Métodos Físicos Aplicados a la Química Orgánica, CONICET, FCEN, UBA). The IR spectra were recorded on a Perkin Elmer Spectrum One FT-IR spectrometer. TLC analyses were carried out on Silica gel $60 \mathrm{~F}_{254}$ using chloroform-methanol (9:1) as solvent. Preparative thin layer separations (PLC) were carried out by centrifugally accelerated, radial chromatography using Chromatotron model 7924T. The rotors were coated with Silica Gel $60 \mathrm{PF}_{254}$ and the layer thickness was $2 \mathrm{~mm}$. Chloroform and increasing percentages of methanol were used as eluent. Reagents, solvents and starting materials were purchased from standard sources and purified according to literature procedures.

Compounds 1a-c were prepared following literature procedure. ${ }^{2}$

\section{Spontaneous transformation of compounds 1a-c}

A typical procedure is described. A solution of 4-hydroxy-2-methyl-1-oxo-1,2dihydroisoquinoline-3-carboxylic acid methyl ester 1a $(0.5 \mathrm{~g})$ freshly prepared in chloroform $(15 \mathrm{~mL})$ was maintained at room temperature for 3-4 weeks until disappearance of the starting product. The crude product showed two main spots by TLC. Separation of the two compounds was achieved by centrifugal PLC. The first band eluted gave 4-hydroxy-2-methyl-1-oxo-1,2dihydroisoquinoline 2 (21-32\%). The structure was confirmed by comparison with an authentic sample prepared by acid hydrolysis of $1 \mathbf{a},{ }^{1 \mathrm{a}} \mathrm{mp}$ and mixed $\mathrm{mp} 225-227^{\circ} \mathrm{C}$. The slower moving band afforded the 4-hydroxy-2-methyl-1,3-dioxo-1,2,3,4-tetrahydroisoquinoline-4-carboxylic acid methyl ester (3a) (40-55\%).

In a manner analogous, spontaneous transformation of $\mathbf{1 b}$ and $\mathbf{1 c}$ afforded compounds $\mathbf{3 b}$ and $\mathbf{3 c}$ respectively.

4-Hydroxy-2-methyl-1,3-dioxo-1,2,3,4-tetrahydroisoquinoline-4-carboxylic acid methyl ester (3a). Yield $38-44 \%, \mathrm{mp} 88-90{ }^{\circ} \mathrm{C}$ (2-propanol). Analyses $(\% \mathrm{C}, \% \mathrm{H}, \% \mathrm{~N})$ calcd for $\mathrm{C}_{12} \mathrm{H}_{11} \mathrm{NO}_{5}: 57.83$, 4.45, 5.62; found: 57.88, 4.50, 5.57; $v_{\max } / \mathrm{cm}^{-1}(\mathrm{KBr}): 3300,3060,1740$, $1732,1596,1250 ; \delta_{\mathrm{H}}$ (DMSO-d6) $8.15\left(1 \mathrm{H}, \mathrm{dd},{ }^{3} J_{8,7}=7.6,{ }^{4} J_{8,6}=1.6, \mathrm{H}-8\right), 7.78\left(1 \mathrm{H}, \mathrm{dt},{ }^{3} J_{6,5 / 6,7}=\right.$ 7.6, $\left.{ }^{4} J_{6,8}=1.6, \mathrm{H}-6\right), 7.64\left(1 \mathrm{H}, \mathrm{t},{ }^{3} J_{7,6 / 7,8}=7.6, \mathrm{H}-7\right), 7.62\left(1 \mathrm{H}, \mathrm{d},{ }^{3} J_{5,6}=7.6, \mathrm{H}-5\right), 7.60(1 \mathrm{H}, \mathrm{ex}, \mathrm{br}$ 
s, OH), $3.61\left(3 \mathrm{H}, \mathrm{s}, \mathrm{OCH}_{3}\right)$ and $3.27\left(3 \mathrm{H}, \mathrm{s}, \mathrm{NCH}_{3}\right) ; \delta_{\mathrm{C}}(\mathrm{DMSO}-\mathrm{d} 6) 170.4(\mathrm{C}-3), 169.1\left(\mathrm{CO}_{2}\right)$, 163.4 (C-1), 137.1 (C-4a), 134.5 (C-6), 129.7 (C-7), 128.1 (C-8), 126.1 (C-5), 124.2 (C-8a), 75.5 (C-4), $53.6\left(\mathrm{OCH}_{3}\right)$ and $27.1\left(\mathrm{NCH}_{3}\right) ; \mathrm{m} / \mathrm{z}(\mathrm{EI}) 250\left(\mathrm{M}^{+}+\mathrm{1}, 2 \%\right), 190\left(\mathrm{M}^{+} \cdot-\mathrm{CO}_{2} \mathrm{CH}_{3}, 100 \%\right)$, $189\left(\mathrm{M}^{+} \cdot-\mathrm{CO}_{2} \mathrm{CH}_{3}-\mathrm{H}, 8 \%\right), 162\left(\mathrm{M}^{+} \cdot \mathrm{CO}_{2} \mathrm{CH}_{3}-\mathrm{CO}, 9 \%\right), 161\left(\mathrm{M}^{+} \cdot-\mathrm{CO}_{2} \mathrm{CH}_{3}-\mathrm{H}-\mathrm{CO}\right.$, 7\%), 149 (25\%), $132\left(\mathrm{M}^{+} \cdot-\mathrm{CO}_{2} \mathrm{CH}_{3}-\mathrm{OCNCH}_{3}, 5 \%\right), 104$ (18\%).

4-Hydroxy-2-methyl-1,3-dioxo-1,2,3,4-tetrahydroisoquinoline-4-carboxylic acid ethyl ester (3b). Yield 44-52\%; mp 77-79 ${ }^{\circ} \mathrm{C}$ (2-propanol). Analyses $(\% \mathrm{C}, \% \mathrm{H}, \% \mathrm{~N})$ calcd for $\mathrm{C}_{13} \mathrm{H}_{13} \mathrm{NO}_{5}$ : 59.31, 4.98, 5.32; found: 59.39, 4.94, 5.36; $v_{\max } / \mathrm{cm}^{-1}(\mathrm{KBr}): 3306,3068,1743,1730,1599 ; \delta_{\mathrm{H}}$ (DMSO-d6) $8.18\left(1 \mathrm{H}, \mathrm{dd},{ }^{3} J_{8,7}=7.7,{ }^{4} J_{8,6}=1.5, \mathrm{H}-8\right), 7.79\left(1 \mathrm{H}, \mathrm{dt},{ }^{3} J_{6,5 / 6,7}=7.7,{ }^{4} J_{6,8}=1.5, \mathrm{H}-6\right)$, $7.66\left(1 \mathrm{H}, \mathrm{t},{ }^{3} J_{7,6 / 7,8}=7.7, \mathrm{H}-7\right), 7.60\left(1 \mathrm{H}, \mathrm{d},{ }^{3} J_{5,6}=7.7, \mathrm{H}-5\right), 7.49(1 \mathrm{H}, \mathrm{ex}$, br s, OH), $4.24(2 \mathrm{H}, \mathrm{q}$, $\left.{ }^{3} J_{\mathrm{CH} 2, \mathrm{CH} 3}=7.2, \mathrm{OCH}_{2}\right), 3.29\left(3 \mathrm{H}, \mathrm{s}, \mathrm{NCH}_{3}\right)$ and $1.29\left(3 \mathrm{H}, \mathrm{t}^{3} J_{\mathrm{CH} 3, \mathrm{CH} 2}=7.2, \mathrm{CH}_{3}\right) ; \delta_{\mathrm{C}}(\mathrm{DMSO}-\mathrm{d} 6)$ 170.0 (C-3), $167.1\left(\mathrm{CO}_{2}\right), 161.2$ (C-1), 136.3 (C-4a), 134.8 (C-6), 129.4 (C-7), 127.9 (C-8), $125.6(\mathrm{C}-5), 124.0(\mathrm{C}-8 \mathrm{a}), 75.8(\mathrm{C}-4), 62.1\left(\mathrm{OCH}_{2}\right), 26.2\left(\mathrm{NCH}_{3}\right)$ and $14.0\left(\mathrm{CH}_{3}\right) ; \mathrm{m} / \mathrm{z}(\mathrm{EI}) 263$ $\left(\mathrm{M}^{+} \cdot+1,1 \%\right), 190\left(\mathrm{M}^{+} \cdot-\mathrm{CO}_{2} \mathrm{C}_{2} \mathrm{H}_{5}, 100 \%\right), 189\left(\mathrm{M}^{+} \cdot-\mathrm{CO}_{2} \mathrm{C}_{2} \mathrm{H}_{5}-\mathrm{H}, 9 \%\right), 162\left(\mathrm{M}^{+} \cdot-\mathrm{CO}_{2} \mathrm{C}_{2} \mathrm{H}_{5}\right.$ $-\mathrm{CO}, 7 \%), 161\left(\mathrm{M}^{+} \cdot-\mathrm{CO}_{2} \mathrm{C}_{2} \mathrm{H}_{5}-\mathrm{H}-\mathrm{CO}, 10 \%\right), 149(23 \%), 132\left(\mathrm{M}^{+}-\mathrm{CO}_{2} \mathrm{C}_{2} \mathrm{H}_{5}-\mathrm{OCNCH}_{3}\right.$, 5\%), $104(19 \%)$.

$\boldsymbol{N}, \boldsymbol{N}$-Diethyl-4-hydroxy-2-methyl-1,3-dioxo-1,2,3,4-tetrahydroisoquinoline-4-carboxamide (3c). Yield 45-56\%; mp 96-98 ${ }^{\circ} \mathrm{C}$ (2-propanol). Analyses $(\% \mathrm{C}, \% \mathrm{H}, \% \mathrm{~N})$ calcd for $\mathrm{C}_{15} \mathrm{H}_{18} \mathrm{~N}_{2} \mathrm{O}_{4}$ : 62.06, 6.25, 9.65; found: 62.15, 6.28, 9.62; $v_{\max } / \mathrm{cm}^{-1}(\mathrm{KBr}): 3310,3050,1746,1731,1600$, $1590 ; \delta_{\mathrm{H}}(\mathrm{DMSO}-\mathrm{d} 6) 8.36\left(1 \mathrm{H}, \mathrm{dd},{ }^{3} J_{8,7}=7.4,{ }^{4} J_{8,6}=1.3, \mathrm{H}-8\right), 7.81\left(1 \mathrm{H}, \mathrm{dt},{ }^{3} J_{6,5 / 6,7}=7.4,{ }^{4} J_{6,8}=\right.$ 1.3, H-6), $7.70\left(1 \mathrm{H}, \mathrm{t},{ }^{3} J_{7,6 / 7,8}=7.4, \mathrm{H}-7\right), 7.66\left(1 \mathrm{H}, \mathrm{d},{ }^{3} J_{5,6}=7.4, \mathrm{H}-5\right), 6.51(1 \mathrm{H}$, ex, br s, OH), 3.75-3.49 (4H, m, $\left.\mathrm{NCH}_{2}\right), 3.45\left(3 \mathrm{H}, \mathrm{s}, \mathrm{NCH}_{3}\right), 1.31\left(3 \mathrm{H}, \mathrm{t}^{3} \mathrm{~J}_{\mathrm{CH} 3, \mathrm{CH} 2}=7.2, \mathrm{CH}_{3}\right)$ and $1.13(3 \mathrm{H}, \mathrm{t}$ $\left.{ }^{3} J_{\mathrm{CH} 3, \mathrm{CH} 2}=7.1, \mathrm{CH}_{3}\right) ; \delta_{\mathrm{C}}(\mathrm{DMSO}-\mathrm{d} 6) 172.1(\mathrm{C}-3), 165.0(\mathrm{CON}), 160.3(\mathrm{C}-1), 137.3(\mathrm{C}-4 \mathrm{a}), 134.4$ (C-6), 129.1 (C-7), 127.5 (C-8), 126.1 (C-5), 124.2 (C-8a), 77.3 (C-4), 41.3 ( $\left.\mathrm{NCH}_{2}\right), 40.9$ $\left(\mathrm{NCH}_{2}\right), 27.0\left(\mathrm{NCH}_{3}\right), 14.1\left(\mathrm{CH}_{3}\right)$ and $12.8\left(\mathrm{CH}_{3}\right) ; \mathrm{m} / \mathrm{z}(\mathrm{EI}) 290\left(\mathrm{M}^{+}, 12 \%\right), 190\left(\mathrm{M}^{+}\right.$$\left.\mathrm{CON}\left(\mathrm{C}_{2} \mathrm{H}_{5}\right)_{2}, 40 \%\right), 162\left(\mathrm{M}^{+} \cdot-\mathrm{CON}\left(\mathrm{C}_{2} \mathrm{H}_{5}\right)_{2}-\mathrm{CO}, 5 \%\right), 149(20 \%), 104$ (17\%), 100 $\left(\mathrm{CON}\left(\mathrm{C}_{2} \mathrm{H}_{5}\right)_{2}{ }^{+}, 60 \%\right), 72\left(\mathrm{~N}\left(\mathrm{C}_{2} \mathrm{H}_{5}\right)_{2}{ }^{+}, 100 \%\right)$.

Isolation of intermediaries in the transformation 1a into 3a. A solution of 4-hydroxy-2methyl-1-oxo-1,2-dihydroisoquinoline-3-carboxylic acid methyl ester 1a $(0.5 \mathrm{~g})$ freshly prepared in chloroform $(15 \mathrm{~mL})$ was maintained at room temperature for approximately 2 weeks until the sample showed by TLC three low $\mathrm{R}_{\mathrm{f}}$ spots besides compounds 1a, $\mathbf{2}$ and 3a. Separation of the compounds was achieved by centrifugal PLC. After the elution of $\mathbf{2}$ and $\mathbf{3 a}\left(\mathrm{HCCl}_{3}-\mathrm{CH}_{3} \mathrm{OH} 9: 1\right)$, the rotor was dried and the low $\mathrm{R}_{\mathrm{f}}$ bands were eluted with DCM under argon atmosphere affording compounds $4 \mathbf{a}, 5 \mathbf{a}$ and $\mathbf{6 a}$

2-Methyl-1,4-dioxo-1,2,3,4-tetrahydroisoquinoline-3-carboxylic acid methyl ester (4a). This compound can not be isolated as a pure oil due to it is easily autooxidable. $v_{\max } / \mathrm{cm}^{-1}(\mathrm{NaCl}$ film): $2957,1724,1641,1585,1445,1323,1247,1006,765 ; \delta_{\mathrm{H}}$ (DMSO-d6) $8.21\left(1 \mathrm{H}, \mathrm{d},{ }^{3} J_{5,6}=7.8, \mathrm{H}-\right.$ 5), 8.01-7.95 (2H, m, H-7 and H-8), $7.90(1 \mathrm{H}, \mathrm{s}, \mathrm{H}-3), 7.86\left(1 \mathrm{H}, \mathrm{t},{ }^{3} J_{6,5 / 6,7}=7.8, \mathrm{H}-6\right), 3.71(3 \mathrm{H}$, $\left.\mathrm{s}, \mathrm{OCH}_{3}\right)$ and $2.95\left(3 \mathrm{H}, \mathrm{s}, \mathrm{NCH}_{3}\right) ; \delta_{\mathrm{C}}(\mathrm{DMSO}-\mathrm{d} 6) 188.7(\mathrm{C}-4), 168.5\left(\mathrm{CO}_{2}\right), 161.4(\mathrm{C}-1), 136.1$ 
(C-7), 132.9 (C-6), 131.9 (C-8a), 129.0 (C-5), 128.2 (C-4a), 126.8 (C-8), 90.8 (C-3), 54.5 $\left(\mathrm{OCH}_{3}\right)$ and $29.2\left(\mathrm{NCH}_{3}\right)$.

3-Hydroperoxy-2-methyl-1,4-dioxo-1,2,3,4-tetrahydroisoquinoline-3-carboxylic acid methyl ester (5a). Yield 9-12\%, mp 150-152 ${ }^{\circ} \mathrm{C}$ (diethyl ether-hexane). This compound presents positive potassium iodide starch test and decomposes slowly on standing affording 3a. Analyses (\%C, $\% \mathrm{H}, \% \mathrm{~N})$ calcd for $\mathrm{C}_{12} \mathrm{H}_{11} \mathrm{NO}_{6}: 54.34,4.18,5.28$; found: $54.24,4.15,5.31 ; v_{\max } / \mathrm{cm}^{-1}(\mathrm{NaCl}$ film): 3215, 2958, 1768, 1707, 1648, 1598, 1582, 1435, 1388, 1300, 1073; $\delta_{\mathrm{H}}\left(\mathrm{DCl}_{3} \mathrm{C}\right) 9.90(1 \mathrm{H}$, ex, br s, OOH), $8.30\left(1 \mathrm{H}, \mathrm{dd},{ }^{3} J_{5,6}=7.6,{ }^{4} J_{5,7}=1.2, \mathrm{H}-5\right), 8.08\left(1 \mathrm{H}, \mathrm{dd},{ }^{3} J_{8,7}=7.6,{ }^{4} J_{8,6}=1.2, \mathrm{H}-8\right)$, $7.84\left(1 \mathrm{H}, \mathrm{dt},{ }^{3} J_{7,6 / 7,8}=7.6,{ }^{4} J_{7,5}=1.2, \mathrm{H}-7\right), 7.72\left(1 \mathrm{H}, \mathrm{dt},{ }^{3} J_{6,5 / 6,7}=7.6,{ }^{4} J_{6,8}=1.2, \mathrm{H}-6\right), 3.83(3 \mathrm{H}, \mathrm{s}$, $\left.\mathrm{OCH}_{3}\right)$ and $3.13\left(3 \mathrm{H}, \mathrm{s}, \mathrm{NCH}_{3}\right) ; \delta_{\mathrm{C}}(\mathrm{DMSO}-\mathrm{d} 6) 185.3(\mathrm{C}-4), 163.7$ and $162.4\left(\mathrm{CO}_{2}\right.$ and $\left.\mathrm{C}-1\right)$, 136.0 (C-7), 133.1 (C-6), 131.2 and 130.0 (C-4a and C-8a), 129.1 (C-5), 126.4 (C-8), 94.6 (C-3), $54.0\left(\mathrm{OCH}_{3}\right)$ and $29.8\left(\mathrm{NCH}_{3}\right) ; \mathrm{m} / \mathrm{z}(\mathrm{EI}) 266\left(\mathrm{M}^{+}+1,3 \%\right), 250\left(\mathrm{M}^{+}+1-\mathrm{O}, 2 \%\right), 232\left(\mathrm{M}^{+}-\right.$ OOH, 32\%), $206\left(\mathrm{M}^{+}-\mathrm{CO}_{2} \mathrm{CH}_{3}, 20 \%\right), 190\left(\mathrm{M}^{+}-\mathrm{CO}_{2} \mathrm{CH}_{3}-\mathrm{O}, 19 \%\right), 176(32 \%), 163(36 \%)$, $162(23 \%), 161(83 \%), 149$ (36\%), 133 (23\%), 132 (34\%), 117 (63\%), 105 (36\%), $104(100 \%)$, 77 (50\%), 76 (83\%).

3-Hydroxy-2-methyl-1,4-dioxo-1,2,3,4-tetrahydroisoquinoline-3-carboxylic acid methyl ester (6a). Yield 10-15\%, mp 118-120 ${ }^{\circ} \mathrm{C}(2$-propanol). Analyses $(\% \mathrm{C}, \% \mathrm{H}, \% \mathrm{~N})$ calcd for $\mathrm{C}_{12} \mathrm{H}_{11} \mathrm{NO}_{5}$ : 57.83, 4.45, 5.62; found: 57.90, 4.48, 5.57; $v_{\max } / \mathrm{cm}^{-1}(\mathrm{KBr}): 3395,2957,1762$, $1703,1670,1598,1433,1301,1239,1081 ; \delta_{\mathrm{H}}\left(\mathrm{DCl}_{3} \mathrm{C}\right), 8.35\left(1 \mathrm{H}, \mathrm{d},{ }^{3} J_{5,6}=7.6, \mathrm{H}-5\right), 8.04(1 \mathrm{H}$, $\left.\mathrm{dd},{ }^{3} J_{8,7}=7.6,{ }^{4} J_{8,6}=1.5, \mathrm{H}-8\right), 7.85\left(1 \mathrm{H}, \mathrm{t},{ }^{3} J_{7,6 / 7,8}=7.6, \mathrm{H}-7\right), 7.73\left(1 \mathrm{H}, \mathrm{dt},{ }^{3} J_{6,5 / 6,7}=7.6,{ }^{4} J_{6,8}=\right.$ 1.5, H-6), $4.89\left(1 \mathrm{H}\right.$, ex, br s, OH), $3.81\left(3 \mathrm{H}, \mathrm{s}, \mathrm{OCH}_{3}\right)$ and $3.29\left(3 \mathrm{H}, \mathrm{s}, \mathrm{NCH}_{3}\right) ; \delta_{\mathrm{C}}(\mathrm{DMSO}-\mathrm{d} 6)$ 188.3 (C-4), $168.5\left(\mathrm{CO}_{2}\right), 161.4(\mathrm{C}-1), 136.1$ (C-7), 132.9 (C-6), 131.9 and 129.0 (C-4a and C8a), 129.0 (C-5), $126.5(\mathrm{C}-8), 86.9(\mathrm{C}-3), 54.4\left(\mathrm{OCH}_{3}\right)$ and $29.1\left(\mathrm{NCH}_{3}\right) ; \mathrm{m} / \mathrm{z}(\mathrm{EI}) 250\left(\mathrm{M}^{+}+1\right.$, $14 \%), 232\left(\mathrm{M}^{+}+1-\mathrm{H}_{2} \mathrm{O}, 6 \%\right), 190\left(\mathrm{M}^{+} \cdot-\mathrm{CO}_{2} \mathrm{CH}_{3}, 100 \%\right), 162\left(\mathrm{M}^{+}-\mathrm{CO}_{2} \mathrm{CH}_{3}-\mathrm{CO}, 29 \%\right), 149$ (66\%), 133 (14\%), 121 (14\%), 105 (20\%), 104 (45\%), 93 (19\%), 76 (40\%), 65 (23\%).

Synthesis of 5a from 1a and conversion to 3a. A mixture of 4-hydroxy-2-methyl-1-oxo-1,2dihydroisoquinoline-3-carboxylic acid methyl ester (1a) (100 $\mathrm{mg}, 0.43 \mathrm{mmol})$ and manganese(III) acetate dihydrate $(11.5 \mathrm{mg}, 0.043 \mathrm{mmol})$ in glacial acetic acid $(10 \mathrm{~mL})$ was stirred at $25^{\circ} \mathrm{C}$ for $2 \mathrm{~h}$ in air, and then the reaction was quenched by adding water $(8 \mathrm{~mL})$. The aqueous reaction mixture was extracted with DCM $(3 \times 9 \mathrm{~mL})$ and the combined extracts were washed with water and with saturated aqueous solution of sodium hydrogencarbonate, dried over anhydrous sodium sulfate and concentrated to dryness. The residue was purified by centrifugal PLC. The obtained hydroperoxide 5a was further recrystallized, yield $76 \%$. Mp and mixed mp with 5a isolated from the spontaneous transformation 1a into 3a: $150-152{ }^{\circ} \mathrm{C}$.

A suspension of $5 \mathbf{a}(100 \mathrm{mg}, 0.38 \mathrm{mmol})$ in water $(5 \mathrm{~mL})$ was heated at reflux for $2 \mathrm{~h}$ affording 3a $(85 \%)$.

Synthesis of 6a from 1a and conversion to 3a. To a cold suspension $\left(0-5^{\circ} \mathrm{C}\right)$ of 4-hydroxy-2methyl-1-oxo-1,2-dihydroisoquinoline-3-carboxylic acid methyl ester (1a) $(100 \mathrm{mg}, 0.43 \mathrm{mmol})$ 
in water $(13 \mathrm{~mL})$, freshly recrystallized NBS $(77 \mathrm{mg}, 0.43 \mathrm{mmol})$ was added. After stirring for 15 minutes, the precipitate was filtered off and washed with cold water. Recrystallization from 2propanol afforded 6a (yield $91 \%$ ). Mp and mixed mp with 6a isolated from the spontaneous transformation 1a into 3a: $118-120^{\circ} \mathrm{C}$. A suspension of $\mathbf{6 a}(100 \mathrm{mg}, 0.40 \mathrm{mmol})$ in water $(5 \mathrm{~mL})$ was heated at reflux for $2 \mathrm{~h}$ affording $\mathbf{3 a}(79 \%)$.

3-Methoxy-2-methyl-1,4-dioxo-1,2,3,4-tetrahydroisoquinoline-3-carboxylic acid methyl ester (7). To a cold suspension $\left(0-5^{\circ} \mathrm{C}\right)$ of 4-hydroxy-2-methyl-1-oxo-1,2-dihydroisoquinoline-3carboxylic acid methyl ester (1a) $(100 \mathrm{mg}, 0.43 \mathrm{mmol})$ in methanol (13 $\mathrm{mL})$, freshly recrystallized NBS (77 mg, $0.43 \mathrm{mmol}$ ) was added. After stirring for 15 minutes, the precipitate was filtered off and washed with cold methanol. Recrystallization from 2-propanol afforded 7. Yield 84\%; mp 136-138 ${ }^{\circ} \mathrm{C}$ (2-propanol). Analyses $(\% \mathrm{C}, \% \mathrm{H}, \% \mathrm{~N})$ calcd for $\mathrm{C}_{13} \mathrm{H}_{13} \mathrm{NO}_{5}: 59.31$, 4.98, 5.32; found: 59.40, 4.95, 5.29; $\nu_{\max } / \mathrm{cm}^{-1}(\mathrm{KBr}): 2956,1773,1698,1661,1598,1429,1377$, $1299,1246,1090 ; \delta_{\mathrm{H}}\left(\mathrm{DCl}_{3} \mathrm{C}\right), 8.37\left(1 \mathrm{H}, \mathrm{dd},{ }^{3} J_{5,6}=7.6,{ }^{4} J_{5,7}=1.2, \mathrm{H}-5\right), 8.09\left(1 \mathrm{H}, \mathrm{dd},{ }^{3} J_{8,7}=7.6\right.$, $\left.{ }^{4} J_{8,6}=1.0, \mathrm{H}-8\right), 7.87\left(1 \mathrm{H}, \mathrm{dt},{ }^{3} J_{7,6 / 7,8}=7.6,{ }^{4} J_{7,5}=1.2, \mathrm{H}-7\right), 7.73\left(1 \mathrm{H}, \mathrm{dt},{ }^{3} J_{6,5 / 6,7}=7.6,{ }^{4} J_{6,8}=1.0\right.$, $\mathrm{H}-6), 3.76\left(3 \mathrm{H}, \mathrm{s}, \mathrm{CO}_{2} \mathrm{CH}_{3}\right), 3.27\left(3 \mathrm{H}, \mathrm{s}, \mathrm{OCH}_{3}\right)$ and $3.08\left(3 \mathrm{H}, \mathrm{s}, \mathrm{NCH}_{3}\right) ; \delta_{\mathrm{C}}(\mathrm{DMSO}-\mathrm{d} 6) 189.0$ $(\mathrm{C}-4), 165.4$ and $162.2\left(\mathrm{CO}_{2}\right.$ and $\left.\mathrm{C}-1\right), 136.1$ (C-7), $133.0(\mathrm{C}-6), 131.6$ and $130.2(\mathrm{C}-4 \mathrm{a}$ and $\mathrm{C}-$ 8a), $129.1(\mathrm{C}-5), 126.3(\mathrm{C}-8), 89.9(\mathrm{C}-3), 53.8\left(\mathrm{OCH}_{3}\right), 51.8\left(\mathrm{OCH}_{3}\right)$ and $28.9\left(\mathrm{NCH}_{3}\right) ; \mathrm{m} / \mathrm{z}(\mathrm{EI})$ $264\left(\mathrm{M}^{+}+1,2 \%\right), 232\left(\mathrm{M}^{+}+1-\mathrm{OCH}_{3}, 12 \%\right), 204\left(\mathrm{M}^{+} \cdot \mathrm{CO}_{2} \mathrm{CH}_{3}, 100 \%\right), 176\left(\mathrm{M}^{+} \cdot-\mathrm{CO}_{2} \mathrm{CH}_{3}-\right.$ CO, 55\%), 163 (99\%), 133 (13\%), 105 (19\%), 104 (66\%), 77 (26\%), 76 (41\%).

4-Methoxy-2-methyl-1,3-dioxo-1,2,3,4-tetrahydroisoquinoline-4-carboxylic acid methyl ester (8). A suspension of $7(100 \mathrm{mg}, 0.38 \mathrm{mmol})$ in methanol $(7 \mathrm{~mL})$ was heated at reflux for $6 \mathrm{~h}$ affording 8. Yield 56\%; mp 98-100 ${ }^{\circ} \mathrm{C}$ (2-propanol). Analyses $(\% \mathrm{C}, \% \mathrm{H}, \% \mathrm{~N})$ calcd for $\mathrm{C}_{13} \mathrm{H}_{13} \mathrm{NO}_{5}$ : 59.31, 4.98, 5.32; found: 59.22, 4.94, 5.28; $v_{\max } / \mathrm{cm}^{-1}(\mathrm{KBr}): 1771,1701,1665$, $1597,1426,1376,1298,1087 ; \delta_{\mathrm{H}}(\mathrm{DMSO}-\mathrm{d} 6) 7.71\left(1 \mathrm{H}, \mathrm{dd},{ }^{3} J_{8,7}=7.5,{ }^{4} J_{8,6}=1.5, \mathrm{H}-8\right), 7.67(1 \mathrm{H}$, $\left.\mathrm{dt},{ }^{3} J_{6,5 / 6,7}=7.5,{ }^{4} J_{6,8}=1.5, \mathrm{H}-6\right), 7.62\left(1 \mathrm{H}, \mathrm{dd},{ }^{3} J_{5,6}=7.5,{ }^{4} J_{5,7}=1.5, \mathrm{H}-5\right), 7.59\left(1 \mathrm{H}, \mathrm{dt},{ }^{3} J_{7,6 / 7,8}=\right.$ $\left.7.5,{ }^{4} J_{7,5}=1.5, \mathrm{H}-.7\right), 3.68\left(3 \mathrm{H}, \mathrm{s}, \mathrm{CO}_{2} \mathrm{CH}_{3}\right), 3.13\left(3 \mathrm{H}, \mathrm{s}, \mathrm{OCH}_{3}\right)$ and $2.93\left(3 \mathrm{H}, \mathrm{s}, \mathrm{NCH}_{3}\right) ; \delta_{\mathrm{C}}$ (DMSO-d6) $169.2\left(\mathrm{CO}_{2}\right), 168.0$ (C-3), 166.1 (C-1), 144.5 (C-4a), 132.9 (C-6), 130.8 (C-7), 123.4 (C-8a), 123.2 (C-5), 123.1 (C-8), $89.1(\mathrm{C}-4), 53.6\left(\mathrm{CO}_{2} \mathrm{CH}_{3}\right), 49.2\left(\mathrm{OCH}_{3}\right)$ and 24.2 $\left(\mathrm{NCH}_{3}\right) ; \mathrm{m} / \mathrm{z}(\mathrm{EI}) 264\left(\mathrm{M}^{+}+1,6 \%\right), 232\left(\mathrm{M}^{+}+1-\mathrm{OCH}_{3}, 17 \%\right), 204\left(\mathrm{M}^{+} \cdot-\mathrm{CO}_{2} \mathrm{CH}_{3}, 84 \%\right), 176$ $\left(\mathrm{M}^{+}\right.$- $\left.\mathrm{CO}_{2} \mathrm{CH}_{3}-\mathrm{CO}, 66 \%\right), 163$ (100\%), 133 (36\%), 105 (51\%), 104 (29\%), 77 (58\%), 76 (71\%), 75 (37\%).

3-Acetoxy-2-methyl-1,4-dioxo-1,2,3,4-tetrahydroisoquinoline-3-carboxylic acid methyl ester (9). To a suspension of 4-hydroxy-2-methyl-1-oxo-1,2-dihydroisoquinoline-3-carboxylic acid methyl ester (1a) (100 mg, $0.43 \mathrm{mmol})$ and magnesium oxide (35 mg, $0.86 \mathrm{mmol})$ in dry DCM $(2 \mathrm{~mL})$ lead(IV) acetate $(189 \mathrm{mg}, 0.43 \mathrm{mmol})$ was added. The reaction mixture was stirred for 3 $\mathrm{h}$ at room temperature and filtered. The filtrate was washed with water, dried and evaporated to dryness. Recrystallization of the residue afforded 9. Yield 78\%; mp 161-163 ${ }^{\circ} \mathrm{C}$ (benzene). Analyses $(\% \mathrm{C}, \% \mathrm{H}, \% \mathrm{~N})$ calcd for $\mathrm{C}_{14} \mathrm{H}_{13} \mathrm{NO}_{6}$ : 57.73, 4.50, 4.81; found: 57.64, 4.53, 4.79; $v_{\max } / \mathrm{cm}^{-1}(\mathrm{KBr}): 3030,2298,1757,1702,1669,1525,1370,1235,1044 ; \delta_{\mathrm{H}}\left(\mathrm{DCl}_{3} \mathrm{C}\right), 8.33(1 \mathrm{H}$, $\left.\mathrm{dd},{ }^{3} J_{5,6}=7.7,{ }^{4} J_{5,7}=1.4, \mathrm{H}-5\right), 8.07\left(1 \mathrm{H}, \mathrm{dd},{ }^{3} J_{8,7}=7.7,{ }^{4} J_{8,6}=1.4, \mathrm{H}-8\right), 7.85\left(1 \mathrm{H}, \mathrm{dt},{ }^{3} J_{7,6 / 7,8}=7.7\right.$, 
$\left.{ }^{4} J_{7,5}=1.4, \mathrm{H}-7\right), 7.81\left(1 \mathrm{H}, \mathrm{dt},{ }^{3} J_{6,5 / 6,7}=7.7,{ }^{4} J_{6,8}=1.4, \mathrm{H}-6\right), 3.77\left(3 \mathrm{H}, \mathrm{s}, \mathrm{OCH}_{3}\right), 3.06(3 \mathrm{H}, \mathrm{s}$, $\left.\mathrm{NCH}_{3}\right)$ and $2.21\left(3 \mathrm{H}, \mathrm{s}, \mathrm{COCH}_{3}\right) ; \delta_{\mathrm{C}}(\mathrm{DMSO}-\mathrm{d} 6) 184.5(\mathrm{C}-4), 168.7,164.9$ and $161.3\left(\mathrm{CO}_{2}\right.$, $\mathrm{COCH}_{3}$ and C-1), 136.0 (C-7), 133.2 (C-6), 132.0 and 131.2 (C-4a and C-8a), 129.0 (C-5), 125.1 (C-8), $87.4(\mathrm{C}-3), 54.0\left(\mathrm{OCH}_{3}\right), 29.9\left(\mathrm{NCH}_{3}\right)$ and $20.8\left(\mathrm{COCH}_{3}\right) ; \mathrm{m} / \mathrm{z}(\mathrm{EI}) 292\left(\mathrm{M}^{+}+1,8 \%\right), 232$ $\left(\mathrm{M}^{+} \cdot-\mathrm{C}_{2} \mathrm{H}_{3} \mathrm{O}_{2}, 47 \%\right), 190\left(\mathrm{M}^{+} \cdot-\mathrm{CO}_{2} \mathrm{CH}_{3}, 100 \%\right), 162\left(\mathrm{M}^{+} \cdot-\mathrm{CO}_{2} \mathrm{CH}_{3}-\mathrm{CO}, 11 \%\right), 149(9 \%), 43$ $(22 \%)$.

A suspension of $9(100 \mathrm{mg}, 0.34 \mathrm{mmol})$ in water was heated at reflux for $5 \mathrm{~h}$ affording $\mathbf{3 a}(74 \%)$. Similarly, a suspension of $9(100 \mathrm{mg}, 0.34 \mathrm{mmol})$ in methanol was heated at reflux for $9 \mathrm{~h}$ affording $8(64 \%)$.

\section{Acknowledgements}

This work was financially supported by the Universidad de Buenos Aires.

\section{References and Notes}

1. (a) Lombardino, J. J. Heterocycl. Chem. 1970, 7, 1057. (b) Lazer, E.; Miao, C. K.; Cywin, C. L.; Sorcek, R.; Wong, H.-C.; Meng, Z.; Potocki, I.; Hoermann, M.; Snow, R. J.; Tschantz, M. A.; Kelly, T. A.; McNeil, D. W.; Coutts, S. J.; Churchill, L.; Graham, V; David, E.; Grob, P. M.; Engel, W.; Meier, H.; Trummlitz, G. J. Med. Chem. 1997, 40, 980. (c) Toyama, M.; Otomasu, H. Chem. Pharm. Bull. 1985, 33, 5543. (d) Beattie, J. F.; Hales, N. J. J. Chem. Soc., Perkin Trans. 1 1992, 751.

2. Blanco, M. M.; Shmidt, M. S.; Schapira, C. B.; Perillo, I. A. Synthesis 2006, 1971.

3. The base peak due to the loss of the 4-substituent is typical of such compounds: Ling, K.-Q.; Ye, J.-H.; Chen, X.-Y.; Ma, D.-J.; Xu, J.-H. Tetrahedron 1999, 55, 9185.

4. Valter, R. É.; Batse, A. É.; Valtere, S. P.Chem. Heterocyclic Comp. 1982, 18,70 and reference 8.

5. Valter, R. É.; Batse, A. É.; Valtere, S. P. Chem. Heterocyclic Comp. 1985, 21, 453.

6. See for example: (a) Rasmussen, C. R. J. Org. Chem. 1974, 39, 1554. (b) E. S. Gould, in Mechanism and Structure in Organic Chemistry, Holt, Rinehart and Winston, New York, N. Y., 1960, pp. 380.

7. (a) Schapira, C. B.; Perillo, I. A. Spectrosc. Lett. 1994, 27, 587 and references cited therein.

(b) Bach, R. D.; Canepa, C. J. Org. Chem. 1996, 61, 6346.

8. Schenker, K. Helv. Chim. Acta 1968, 51, 413.

9. Felner, I.; Schenker K. Helv. Chim. Acta 1969, 52, 1810.

10. Beattie, J. F.; Hales, N. J. J. Chem. Soc., Perkin Trans. 1 1992, 751.

11. Alonso-Silva, I. J.; Pardo, M.; Soto, J. L. Heterocycles 1988, 27, 357. 
12. This type of reaction represents a particular case of C-3 oxidative functionalyzation of 4hydroxy-isoquinolinones. Other examples employ bromo ${ }^{13}, \mathrm{NBS}^{11,13}$ and thionyl chloride ${ }^{10}$. In all cases the keto form is considered as the reactive species.

13. Ben-Ishai, D.; Inbal, Z.; Warshawsky, A. J. Heterocycl. Chem. 1970, 7, 621.

14. Ozols, J.; Liepins, E.; Mazeika, I.; Vigante, B.; Duburs, G. Khim. Geterotsikl Soedin. 1978, 789; Chem. Abstr. 1978, 89, 109014.

15. Among others: (a) March, J. In Advanced Organic Chemistry, Wiley Interscience: New York, $5^{\text {th }}$ Edition, 2001, pp 920-922. (b) Ingold K. U. Acc. Chem. Res. 1969, 2, 1. (c) Mayo, F. R. Acc. Chem. Res. 1968, 1, 193.

16. (a) Sharp, D. B; Miller, E. L. J. Am. Chem. Soc. 1952, 74, 5643. (b) Selman, S.; Eastham, J. F.Quart. Rev. 1960, 14, 221.

17. (a) Klásek, A.; Kořistec, K.; Polis, J.; Košmrlj, J. Tetrahedron 2000, 56, 1551. (b) S. Kafka, J.; Klásek, A.; Košmrlj, J. J. Org. Chem. 2001, 66, 6394.

18. Sprecher, M. Chemtracts: Org. Chem. 1991, 4, 307.

19. Bachi, M. D.; Bosch, E. Heterocycles 1989, 28, 579.

20. Jeffrey, G. A. in An Introduction to Hydrogen Bonding, Oxford University Press, Oxford, NY, 1982 pp. 98-103.

21. Similar results were observed in related compounds: Perillo, I. A.; Kremenchuzky, L. D.; Blanco, M. M. J. Mol. Struct. 2009, 921, 307 and references cited therein.

22. Giacomelli, L.; Santo, M.; Cattana, R.; Silber, J. J.; Blanco, M. M.; Levin, G.; Perillo, I. A. XIV SINAQO, Rosario, Argentina, November 9-12, 2003: Abstract N SQO 44.

23. Compounds of this family are obtained by reaction of 1,3-isoquinolinediones with singlet oxygen. ${ }^{3}$ 